Draft Version July 18, 2008

Preprint typeset using $\mathrm{LATEX}_{\mathrm{E}}$ style emulateapj v. 10/09/06

\title{
THE BIERMANN BATTERY IN COSMOLOGICAL MHD SIMULATIONS OF POPULATION III STAR FORMATION
}

\author{
Hao Xu ${ }^{1,2}$, Brian W. O’Shea ${ }^{2,3}$, David C. Collins ${ }^{1}$, Michael L. Norman ${ }^{1}$, Hui Li $^{2}$, And Shengtai Li ${ }^{2}$ \\ Draft version July 18, 2008
}

\begin{abstract}
We report the results of the first self-consistent three-dimensional adaptive mesh refinement magnetohydrodynamical simulations of Population III star formation including the Biermann Battery effect. We find that the Population III stars formed including this effect are both qualitatively and quantitatively similar to those from hydrodynamics-only (non-MHD) cosmological simulations. We observe peak magnetic fields of $\simeq 10^{-9} \mathrm{G}$ in the center of our star-forming halo at $z \simeq 17.55$. The magnetic fields created by the Biermann Battery effect are predominantly formed early in the evolution of the primordial halo at low density and large spatial scales, and then grow through compression and by shear flows. The fields seen in this calculation are never large enough to be dynamically important (with $\beta \geq 10^{15}$ at all times), and should be considered the minimum possible fields in existence during Population III star formation, and may be seed fields for the stellar dynamo or the magnetorotational instability at higher densities and smaller spatial scales.

Subject headings: cosmology: theory - galaxies: high-redshift - magnetohydrodynamics - stars: formation
\end{abstract}

\section{INTRODUCTION}

The nature of the first generation of stars, as well as their influence on later structure formation, is a fundamental problem in cosmology. A great deal of theoretical progress has been made (see recent reviews by Bromm \& Larson (2004), Glover (2005), and Ciardi \& Ferrara (2005). In the past decade, cosmological hydrodynamic simulations of Population III star formation have achieved great success, and significantly different numerical methods have produced results that agree quite well (Abel et al. 2002; Bromm et al. 2002; Yoshida et al. 2003; O'Shea \& Norman 2007). These calculations have given a reasonably clear picture of the formation process of Population III stars, and have provided some constraints on many of their important properties.

These calculations, while useful, largely ignore an important issue: the relevance of magnetic fields in Population III star formation. Magnetic fields are widely observed in our galaxy, in other galaxies, and in galaxy clusters, and the origin of these fields is one of the most fundamental and challenging problems in astrophysics (Carilli \& Taylor 2002; Widrow 2002). One possibility is that magnetic fields are created and amplified in the first generation of stars and are spread throughout the IGM when these stars explode, providing seed fields for later generations of stars and for further amplification by dynamo effects. If some seed magnetic fields exist before Population III stars form, they may help to remove angular momentum from the star-forming clouds, significantly changing the ultimate mass range of these stars (Pudritz \& Silk 1989; Davies \& Widrow 2000).

Several groups have examined the importance of mag-

\footnotetext{
Electronic address: haxu@ucsd.edu

${ }^{1}$ Center for Astrophysics and Space Sciences, University of California, San Diego, 9500 Gilman Drive, La Jolla, CA 92093

2 Theoretical division, Los Alamos National Lab, Los Alamos, NM 87545

${ }^{3}$ Department of Physics and Astronomy and Lyman Briggs College, Michigan State University, East Lansing, MI 48824
}

netic fields on the evolution of Population III protostellar disks using analytic or semi-analytic models. These include Silk \& Langer (2006), Tan \& McKee (2004) and Tan \& Blackman (2004), who model (among other aspects of primordial star formation and evolution) dynamos in primordial accretion disks. Other authors, including Flower \& Pineau des Forêts (2003) and Maki \& Susa (2007), use one-zone calculations to examine the collapse of the primordial star-forming cloud and the assumptions of flux-freezing. While useful, these models do not self-consistently include the effects of both magnetic fields and cosmological structure formation.

In this Letter, we report the results of the first magnetohydrodynamic simulations of Population III star formation including the Biermann Battery effect (Biermann 1950) within the context of cosmological structure formation. We describe our numerical methods and the simulation used in Section 2, present our key results in Section 3, and discuss and summarize our results in Section 4.

\section{METHODOLOGY}

The simulation discussed in this Letter was performed using the adaptive mesh N-body plus hydrodynamics code Enzo (O'Shea et al. 2004; Norman et al. 2007), which has recently been extended to including the equations of ideal magnetohydrodynamics. The MHD solver is a high-order Godunov-type finite-volume numerical solver ( Li \& Li 2003; Li 2005; Li et al. 2008). This solver was recently successfully used to study magnetic jets (Li et al. 2006; Nakamura et al. 2006, 2007). To maintain the divergence-free magnetic field condition, we used a constrained transport (CT) scheme (Balsara \& Spicer 1999) as well as a modified divergence-free reconstruction scheme original proposed by Balsara (2001) that includes second-order-accurate divergence-free restriction and prolongation for magnetic fields in the adaptive mesh hierarchy (Collins \& Norman 2008). These equations are solved in cosmological calculations in the comov- 
ing frame, as described in $\mathrm{Xu}$ et al. (2008). To track the pressure more accurately in hypersonic regions, we have implemented the modified entropy equation given in Ryu et al. (1993) and the internal energy equation given in Bryan et al. (1995) in our code.

To study the effect of the Biermann battery during the formation of Population III stars, the battery term is added to the induction equation (Kulsrud 2005):

$$
\begin{aligned}
\frac{\partial \mathbf{B}}{\partial t} & =\nabla \times(\mathbf{v} \times \mathbf{B})+\frac{\mathbf{c} \nabla \mathbf{p}_{\mathbf{e}} \times \nabla \mathbf{n}_{\mathbf{e}}}{\mathbf{n}_{\mathbf{e}}^{\mathbf{e}}} \\
& =\nabla \times(\mathbf{v} \times \mathbf{B})+\frac{\mathbf{c m}}{\mathbf{e}} \frac{\mathbf{1}}{\mathbf{1}+\chi} \frac{\nabla \mathbf{p} \times \nabla \rho}{\rho^{2}}
\end{aligned}
$$

where $c$ is speed of light, $p_{e}$ is the electron pressure, $n_{e}$ is the electron number density, $e$ is the electron charge, $m$ is the average mass per particle and $\chi$ is the ionization fraction. This term is non-zero in regions where curved shocks are formed. The second equation assumes that $m$ and $\chi$ are unchanged in space, which is a reasonable approximation locally. As pointed out by Kulsrud et al. (1997), the ionization fraction enters into the induction equation through $1+\chi$, so even a very small ionization fraction is enough to generate magnetic fields. In our simulation, we took $m=1.2 m_{H}$, where $m_{H}$ is the mass of a neutral hydrogen atom. Note that the electron fraction is $\ll 1$ at all times in this star formation scenario, so we set $\chi=0$ rather than calculating the electron fraction in every cell. Given the approximation above, this introduces negligible error. The battery term is added to the simulation through the EMF term in the constrained transport algorithm, ensuring that no divergence of magnetic fields is introduced to the system.

Since the electron fraction declines rapidly at the halo core, ambipolar diffusion may be important in star formation simulation (Abel et al. 2002). To address this issue, we compared the ambipolar diffusion timescale with the dynamic timescale in our simulation, as was done by (O'Shea \& Norman 2007). The ratio is always larger than 10 over the density range of interest, suggesting that ambipolar diffusion does not play a significant role up to the densities that we study.

Our simulation setup is similar to that described in O'Shea \& Norman (2007). We use a N-body plus hydrodynamics simulation with a comoving box size of 0.3 $\mathrm{h}^{-1} \mathrm{Mpc}$, initialized at $z=99$ with an Eisenstein \& $\mathrm{Hu}$ power spectrum (Eisenstein \& Hu 1999), and with cosmological parameters $\Omega_{b}=0.04, \Omega_{m}=0.3, \Omega_{\Lambda}=0.7$, $h=0.7, \sigma_{8}=0.9$, and $n_{s}=1.0$. The simulation was first run with a $128^{3}$ root grid and three levels of AMR to $z=15$, where the most massive halo in the volume was found. The simulation was then re-centered on this halo, using a $128^{3}$ root grid and three levels of static nested grids, giving a dark matter and baryon resolution of $1.81 \mathrm{~h}^{-1} \mathrm{M}_{\odot}$ and $0.28 \mathrm{~h}^{-1} \mathrm{M}_{\odot}$, respectively, and an initial comoving spatial resolution of $293 \mathrm{~h}^{-1} \mathrm{pc}$. The simulation was started at $z=99$, initialized with zero magnetic fields throughout, and allowed to run with a maximum of 22 levels of adaptive mesh refinement until the collapse of the most massive halo, at $z \simeq 17.55$, using the nine-species molecular chemistry of Abel et al. (1997) and Anninos et al. (1997), but modified for high densities as described in Abel et al. (2002). Further details of the simulation setup and physics can be found in
O'Shea \& Norman (2007).

\section{RESULTS}

Over the course of the simulation, magnetic fields are produced at various physical scales by the Biermann Battery process. Figure 1 shows the evolution of sphericallyaveraged radial profiles of the baryon number density and magnetic field strength of the primordial star-forming halo at several times during the halo evolution. Since the magnetic fields are very small $\left(\beta \equiv P_{\text {thermal }} / P_{\text {magnetic }}\right.$ is greater than $10^{15}$ at all times), the gas collapse at the halo core is almost the same as the hydrodynamic $(B=$ 0 ) case described by O'Shea \& Norman (2007). The profiles start at $z=40$, where the magnetic fields generated by the Biermann Battery are of order $10^{-18} \mathrm{G}$, which is consistent with theoretical predictions (Pudritz \& Silk 1989; Davies \& Widrow 2000). In plasmas with a large beta $(\gg 1)$ and large mass-to-flux ratio, magnetic fields follow the gas falling into the core passively, so after being generated at low densities, the magnetic fields are primarily amplified by being carried along with collapsing baryons. This is shown more clearly in Figure 2, and will be discussed in more detail later. At low density (up to $\mathrm{n}_{b} \simeq 2 \mathrm{~cm}^{-3}$, corresponding to the evolution of the gas up to $z \sim 20$ ), the Biermann Battery is effective at creating magnetic fields. The central magnetic field scales with density as $|B| \propto \rho$, suggesting that the magnetic field is enhanced by both the Biermann Battery effect and by gas collapse. At higher densities (after $z \simeq 20$ ), the magnetic field at the center of the halo is amplified as $|B| \propto \rho^{2 / 3}$, as expected in spherical collapse. At later times, when the gas in the center of the halo collapses, the magnetic field in the center of the halo rises to $\sim 10^{-9} \mathrm{G}$ as the central number density grows to $\mathrm{n}_{b} \simeq 10^{10} \mathrm{~cm}^{-3}$.

It is useful to examine the history of magnetic field amplification inside the halo in more detail. This is shown in Figure 2, which shows the mass- and volume-weighted magnetic fields inside the halo virial radius as a function of redshift (top panel), and also shows the mass-weighted mean magnetic field strength in the inner $100 \mathrm{M}_{\odot}$ of gas as a function of the mass-weighted baryon number density of gas in the same Lagrangian region (bottom panel). From these two plots, the two stages of magnetic field amplification can be observed. From $z=40$ to $z=20$, the magnetic fields slowly increase to $10^{-15} \mathrm{G}$. At this stage, the mass-weighted and volume-weighted fields are quite similar, because the magnetic fields are created and amplified by the Biermann Battery at large spatial scales, and the fields are distributed uniformly by mergers and shear flows (see Roettiger et al. (1999)). During the second stage of evolution, after $z=20$, the volumeweighted and mass-weighted fields evolve in very different ways, with the mass-weighted magnetic field growing rapidly along with the increasing baryon density in the halo cores.

The bottom panel of Figure 2 shows that at late times (high densities), the magnetic field in the core of the halo increases as $|B| \propto \rho^{2 / 3}$, or solely from field amplification due to magnetic fields frozen into spherically-contracting gas. The collapse timescale at the center of the halo is proportional to the cooling time, which is quite short at high densities, making any significant magnetic field creation by the Biermann Battery mechanism difficult in the halo center. The baryon density in the halo core 

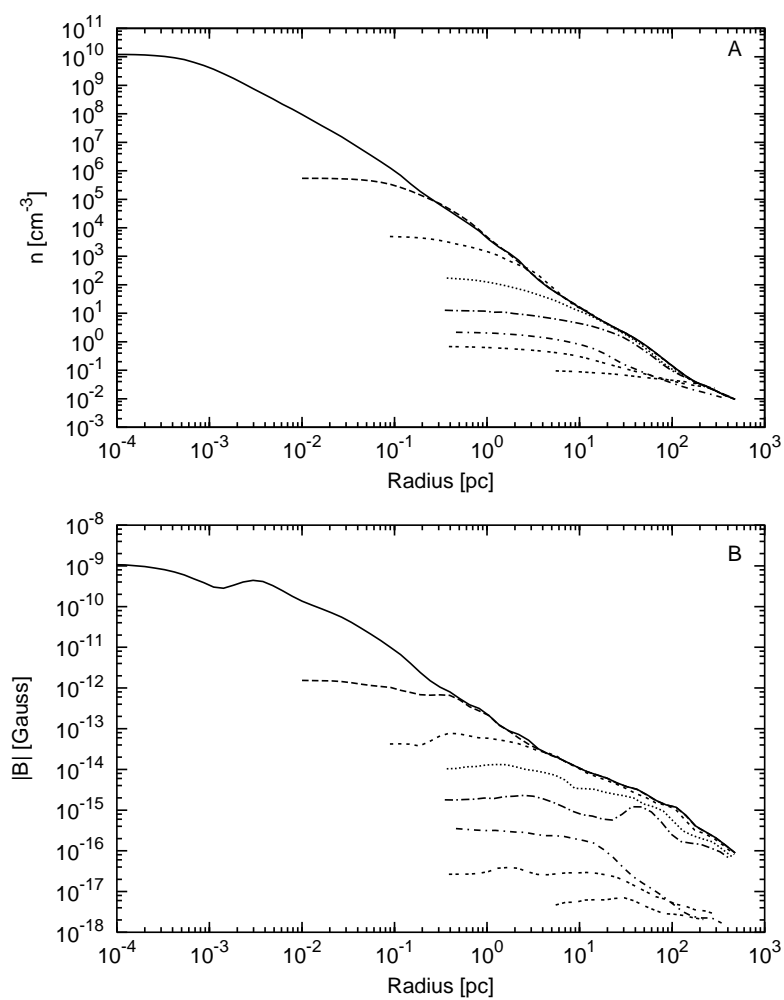

FIG. 1.- Evolution of spherically-averaged, mass-weighted radial profiles of baryon number density (top) and magnetic field strength (bottom) of the Population III star-forming halo. Lines correspond to (from bottom to top in each panel) $z=$ $40,30,25,20,19,18,17.61,17.55$.

grows about 9 orders of magnitude (from 1 to $10^{10} \mathrm{~cm}^{-3}$ between $z=20$ and $z=17.55$, when the simulation is terminated), and the magnetic fields in the core increase by about 5 orders of magnitude (from $10^{-15}$ to $10^{-9}$ $\mathrm{G})$. Though the core region contains less than one percent of the halo gas, the mass-weighted magnetic field in the halo increases by approximately four orders of magnitude, from $\sim 10^{-15}$ to $10^{-11} G$, between $\mathrm{z}=20$ and $\mathrm{z}=17.55$.

To show where magnetic field generation via the Biermann Battery takes place, and to provide a more quantitative understanding of its effect in different stages of halo evolution, we show in Figure 3 the two-dimensional distribution of the instantaneous magnetic field generation rate via the Biermann Battery versus baryon overdensity at four simulation outputs from $z=40$ to $z=17.55$. At early times, very little gas is at high overdensities, and the magnetic field generation rate is below $10^{-35} \mathrm{G} / \mathrm{s}$. The total time taken during the simulation, from $z=99$ to $z=17.55$, is approximately $6.2 \times 10^{15}$ seconds. If the magnetic fields are only created by the Biermann Battery without any amplification from shear flows or collapse, the magnetic fields would be at most $10^{-19} \mathrm{G}$ at $z \sim 18$. By $z=40\left(\sim 1.5 \times 10^{15}\right.$ seconds, or $5 \times 10^{7}$ years, after the beginning of the calculation), fields reach a strength of $10^{-18} \mathrm{G}$, suggesting that some amplification is taking place through gas collapse. While the rate of magnetic field generation via the Biermann Battery can actually be much higher at high overdensity (as seen in the bottom right panel, at $z=17.55$ ), the rate never exceeds $10^{-30} \mathrm{G} / \mathrm{s}\left(\sim 3 \times 10^{-23} \mathrm{G} / \mathrm{yr}\right)$. Given that the collapse of the core, from a peak baryon
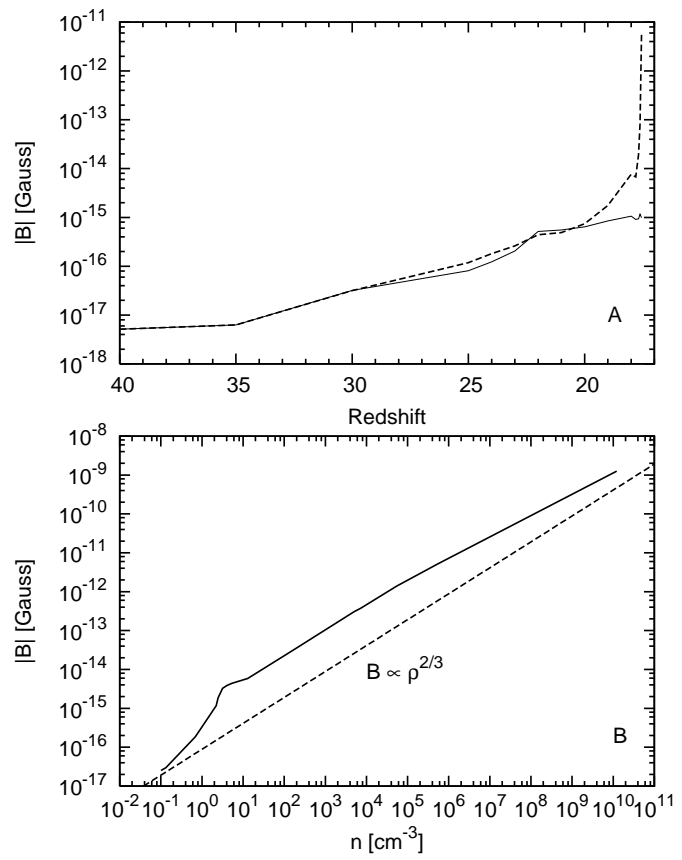

FIG. 2. - Top panel: mass-weighted (dashed line) and volumeweighted (solid line) mean magnetic field strength inside the virial radius of the collapsing halo as a function of redshift. Bottom panel: mass-weighted mean magnetic field strength as a function of baryon number density inside the core of the primordial star forming halo at the final simulation output (solid line; $\mathrm{M}_{e n c} \simeq$ $100 \mathrm{M}_{\odot}$ ). The dashed line shows $|B| \propto \rho^{2 / 3}$ (as expected by flux freezing and spherical collapse).
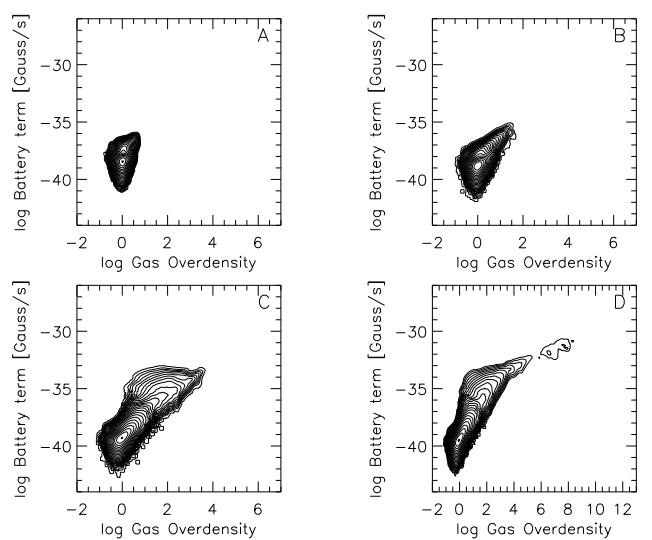

FIG. 3.- Mass-weighted two-dimensional distributions of the instantaneous rate of magnetic field generation via the Biermann battery vs. baryon overdensity, at $z=40$ (top left), $z=30$ (top right), $z=20$ (bottom left) and $z=17.55$ (bottom right). Each panel includes all gas within the virial radius at a given redshift. Contour lines are logarithmic, and show relative values between the highest and lowest non-zero values in each panel.

density of $\mathrm{n}_{b} \sim 1 \mathrm{~cm}^{-3}$ to $10^{10} \mathrm{~cm}^{-3}$, takes approximately $10^{14}$ seconds $\left(\simeq 3 \times 10^{6}\right.$ years $)$, magnetic fields larger than $10^{-16} \mathrm{G}$ cannot be made with the Biermann Battery during this time, strongly suggesting that the battery effect makes little contribution to magnetic field amplification during the final collapse.

\section{DISCUSSION AND CONCLUSIONS}

In this Letter, we have shown results from the first cosmological magnetohydrodynamical simulation of Population III star formation including the Biermann Battery effect. This effect is one of the most robust methods of generating magnetic fields in the Universe (Biermann 
1950), and thus provides useful constraints on the minimum magnetic field expected in situations where Population III star formation will take place.

We find from our simulation that small magnetic fields are primarily generated via the Biermann Battery at relatively low overdensities, and are then amplified to values of nearly $10^{-9} \mathrm{G}$ at the center of the cosmological halo via gravitational collapse. While significant, this magnetic field is still quite small - the plasma $\beta$ is never smaller than $10^{15}$ at any point during the simulation. This suggests strongly that the magnetic fields do not play a significant dynamical role up to $\mathrm{n}_{B} \sim 10^{10} \mathrm{~cm}^{-3}$, when the simulation is terminated. As a result, the evolution of the primordial gas within the cosmological halo is very close to the hydrodynamic results obtained by O'Shea \& Norman (2007). At later times, however, the magnetic fields may be amplified to dynamically relevant values via a process such as the magnetorotational instability or a dynamo (Silk \& Langer 2006; Tan \& McKee 2004; Tan \& Blackman 2004).

The simulation of further evolution of our evolving protostellar core is currently beyond the capability of our numerical tools. However, there are multiple avenues that we can follow to more thoroughly explore the relevance of magnetic fields in Population III star formation. We can begin our calculation with seed fields having strengths based on limits from observation and theory (Widrow 2002). We can examine the effects of magnetic fields at higher densities by implementing more chemical and cooling processes (as discussed by Turk et al. 2008). Both of these projects are underway, and we will report on the results in an upcoming paper.
To summarize, we have performed the first calculations that incorporate the Biermann Battery in cosmological magnetohydrodynamic simulations of Population III star formation. Our key results are as follows:

1. From an initial state with no magnetic fields, a combination of the Biermann Battery and compressional amplification can result in fields with strengths of $|B| \simeq$ $10^{-9} \mathrm{G}$ at $\mathrm{n}_{B} \simeq 10^{10} \mathrm{~cm}^{-3}$ at the center of a cosmological halo where a Population III star will form.

2. The Biermann Battery creates fields predominantly at low density $\left(\mathrm{n}_{B} \leq 10 \mathrm{~cm}^{-3}\right)$ and large spatial scales in Population III star-forming halos.

3. The magnetic fields created by the Biermann Battery are dynamically unimportant at all densities below $\mathrm{n}_{B} \simeq 10^{10} \mathrm{~cm}^{-3}-\beta \equiv P_{t h} / P_{B} \geq 10^{15}$ at all times during the evolution of the halo.

We would like to thank Tom Abel, Greg Bryan, Mordecai-Mark Mac Low, Matthew Turk, and Dan Whalen for useful conversations. HX and BWO are supported by IGPP at Los Alamos National Laboratory, and carried out this work under the auspices of the National Nuclear Security Administration of the U.S. Department of Energy at Los Alamos National Laboratory under Contract No. DE-AC52-06NA25396. HX and MLN acknowledge partial support from NSF grant AST-0708960. The simulation described in this paper was performed at the San Diego Supercomputing Center with computing time provided by NRAC allocation MCA98N020.

\section{REFERENCES}

Abel, T., Anninos, P., Zhang, Y., \& Norman, M. L. 1997, New Astronomy, 2, 181

Abel, T., Bryan, G. L., \& Norman, M. L. 2002, Science, 295, 93

Anninos, P., Zhang, Y., Abel, T., \& Norman, M. L. 1997, New Astronomy, 2, 209

Balsara, D. S. 2001, Journal of Computational Physics, 174, 614

Balsara, D. S., \& Spicer, D. S. 1999, Journal of Computational Physics, 149, 270

Biermann, L. 1950, Z. Naturforsch., 5

Bromm, V., Coppi, P. S., \& Larson, R. B. 2002, ApJ, 564, 23

Bromm, V., \& Larson, R. B. 2004, ARA\&A, 42, 79

Bryan, G. L., Norman, M. L., Stone, J. M., Cen, R., \& Ostriker, J. P. 1995, Comp. Phys. Comm, 89, 149

Carilli, C. L., \& Taylor, G. B. 2002, ARA\&A, 40, 319

Ciardi, B., \& Ferrara, A. 2005, Space Science Reviews, 116, 625

Collins, D., \& Norman, M. 2008, in preparation

Davies, G., \& Widrow, L. M. 2000, ApJ, 540, 755

Eisenstein, D. J., \& Hu, W. 1999, ApJ, 511, 5

Flower, D. R., \& Pineau des Forêts, G. 2003, MNRAS, 341, 1272

Glover, S. 2005, Space Science Reviews, 117, 445

Kulsrud, R. M. 2005, Plasma physics for astrophysics (Plasma physics for astrophysics / Russell M. Kulsrud. Princeton, N.J. : Princeton University Press, c2005. (Princeton series in astrophysics))

Kulsrud, R. M., Cen, R., Ostriker, J. P., \& Ryu, D. 1997, ApJ, 480, 481

Li, H., Lapenta, G., Finn, J. M., Li, S., \& Colgate, S. A. 2006, ApJ, 643,92
Li, S. 2005, Journal of Computational Physics, 203, 344

Li, S., \& Li, H. 2003, Los Alamos Technical Report

Li, S., Li, H., \& Cen, R. 2008, ApJS, 174, 1

Maki, H., \& Susa, H. 2007, PASJ, 59, 787

Nakamura, M., Li, H., \& Li, S. 2006, ApJ, 652, 1059

-. 2007, ApJ, 656, 721

Norman, M. L., Bryan, G. L., Harkness, R., Bordner, J., Reynolds, D., O'Shea, B., \& Wagner, R. 2007, ArXiv e-prints, 705

O'Shea, B., Bryan, G., Bordner, J., Norman, M., Abel, T., \& Harkness, R. amd Kritsuk, A. 2004, Adaptive Mesh Refinement - Theory and Applications, ed. T. Plewa, T. Linde, \& G. Weirs (Springer-Verlag)

O'Shea, B. W., \& Norman, M. L. 2007, ApJ, 654, 66

Pudritz, R. E., \& Silk, J. 1989, ApJ, 342, 650

Roettiger, K., Stone, J. M., \& Burns, J. O. 1999, ApJ, 518, 594

Ryu, D., Ostriker, J. P., Kang, H., \& Cen, R. 1993, ApJ, 414, 1

Silk, J., \& Langer, M. 2006, MNRAS, 371, 444

Tan, J. C., \& Blackman, E. G. 2004, ApJ, 603, 401

Tan, J. C., \& McKee, C. F. 2004, ApJ, 603, 383

Turk, M. J., Abel, T., \& O'Shea, B. W. 2008, in American Institute of Physics Conference Series, Vol. 990, First Stars III, 16-20

Widrow, L. M. 2002, Reviews of Modern Physics, 74, 775

Xu, H., Li, H., Collins, D., Li, S., \& Norman, M. L. 2008, ApJ, 681, L61

Yoshida, N., Abel, T., Hernquist, L., \& Sugiyama, N. 2003, ApJ, 592,645 